\title{
Camera-to-Camera Mapping for Hybrid Pan-Tilt-Zoom Sensors Calibration
}

\author{
Julie Badri ${ }^{1,2}$, Christophe Tilmant ${ }^{1}$, Jean-Marc Lavest ${ }^{1}$, Quonc-Cong Pham ${ }^{2}$, \\ and Patrick Sayd ${ }^{2}$ \\ ${ }^{1}$ LASMEA, Blaise Pascal University, \\ 24 avenue des Landais, Aubiere, F-63411 France \\ ${ }^{2}$ CEA, LIST, \\ Boîte Courrier 65, Gif-sur-Yvette, F-91191 France \\ \{julie.badri, christophe.tilmant, jean-marc.lavest\} @univ-bpclermont.fr, \\ $\{$ quoc-cuong.pham, patrick. sayd $@$ @cea.fr
}

\begin{abstract}
Video surveillance becomes more and more extended in industry and often involves automatic calibration system to remain efficient. In this paper, a video-surveillance system that uses stationary-dynamic cameras devices is presented. The static camera is used to monitor a global scene. When it detects a moving object, the Pan-Tilt-Zoom (PTZ) camera is controlled to be centered on this object. We describe a method of camera-to-camera calibration, integrating zoom calibration in order to command the angles and the zoom of the PTZ camera. This method enables to take into account the intrinsic camera parameters, the $3 \mathrm{D}$ scene geometry and the fact that the mechanism of inexpensive camera does not fit the classical geometrical model. Finally, some experiment results attest the accuracy of the proposed solution.
\end{abstract}

Keywords: Camera-to-camera calibration, zoom calibration, visual servoing, Pan-Tilt-Zoom camera, video surveillance.

\section{Introduction}

Surveillance companies want simultaneously to monitor a wide area with a limited camera network and to record identifiable imagery of all the people passing through that area. To solve this problem, it has been proposed to combine static cameras having a large field-of-view with Pan-Tilt-Zoom cameras. Indeed, it is possible to control the angle of rotation of the PTZ camera (pan and tilt angles) and the zoom. In practice, the system proceeds as follows. A scene event as a moving subject is detected and located using the static camera. The PTZ camera must be controlled with the information of the static camera in order to adjust its pan, tilt and zoom such as the object of interest remains in the field of view. Then, the high resolution image can be recorded in order to apply face or gesture recognition algorithm, for example.

The main problem to solve in this system is how to control the PTZ camera parameters from the information of the object position extracted in the static

B.K. Ersbøll and K.S. Pedersen (Eds.): SCIA 2007, LNCS 4522, pp. 132 141 2007.

(C) Springer-Verlag Berlin Heidelberg 2007 
camera. These last years, two approaches emerged. Either, each camera is calibrated in order to obtain the intrinsic and extrinsic camera parameters before to find a general relation between $2 \mathrm{D}$ coordinates in the static camera and the pan and tilt angles, like Horaud et al. [5] and Jain et al. [6] or cameras are not calibrated like Zhou et al. 12 and Senior et al. 9. They learned a look-uptable (LUT) linking several positions in the static camera with the corresponding pan-tilt angles. Then, for another point, they estimate the corresponding pan-tilt angles by interpolating using the closest learned values.

In order to position the presented paper, we develop the existing works. In the first case, wthe problem camera calibration, and in particular dynamic camera calibration has been extensively addressed. Either cameras calibration is based on the fact that it is a stereo-vision system like Horaud et al. 5] or each camera is calibrated separately like Jain et al. 6]. In this case, most existing methods for calibrating a pan-tilt camera suppose simplistic geometry model of motion in which axes of rotation are orthogonal and aligned with the optical center (1), [2], 10], 4], 11]). If this assumptions can be suitable for expensive mechanisms, they are not to model the true motion of inexpensive pan-tilt mechanisms. In reality a single rotation in pan rotation induces a curved displacement in the image instead of straight lines.

Recently, Jain et al. 6] proposed a new calibration method with more degrees of freedom. As with other methods the position and orientation of the camera's axes can be calibrated, but it can be also calibrated the rotation angle. It is more efficient, more accurate and less computationally expensive than the previous works. Actually, Jain et al. [6] mean to be the only one to propose a method without simplistic hypothesis. The calibration step involves the presence of a person to deal with the calibration marks. So, this method can not be used in the goal of a turnkey solution for a no-expert public.

Now, methods based on the no-direct camera calibration are focused. Few people have explored this approach. The first were Zhou et al. [12] who used collocated cameras whose viewpoints are supposed to be identical. The procedure consisted of collecting a series of pixel location in the stationary camera where a surveillance subject could later appear. For each pixel, the dynamic camera was manually moved to center the image on the subject. The pan and tilt angles were recorded in a LUT indexed by the pixel coordinates in the stationary camera. Intermediate pixels in the stationary camera were obtained by a linear interpolation. At run time, when a subject is located in the stationary camera, the centering maneuver of dynamic camera used the recorded LUT. The advantage of this approach is that calibration marks are not used. This method is based on the 3D information of the scene but the LUT is learned manually.

More recently, Senior et al. 9] proposed a procedure more automatic than Zhou et al. [12. To steer the dynamic camera, they need to know a sequence transformations to enable to link a position with the pan-tilt angles. This transformations are adapted to pedestrian tracking. An homography links the foot position of the pedestrian in the static camera with the foot position in the dynamic camera. A transformation links the foot position in the dynamic 

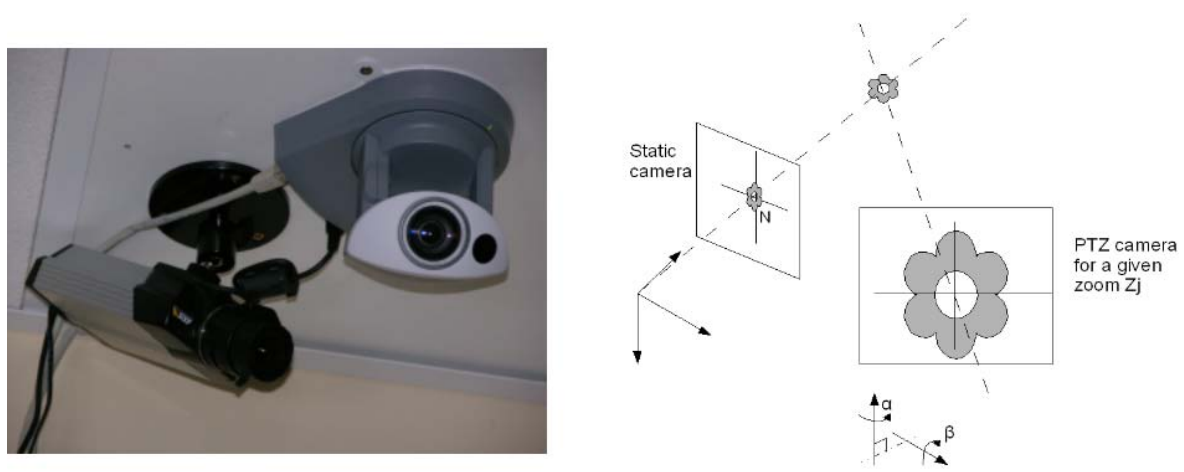

Fig. 1. Our system of collocated cameras : the static camera is on the left and the dynamic camera is on the right

camera with the position in the dynamic camera. Finally, a third transformation encoded in a LUT as Zhou et al. 12] links the head position in the dynamic camera with pan-tilt angles. These transformations are learned automatically from unlabelled training data. The default of this method is the step of the establishment of the training data. If this method is used for a turnkey solution for a no-expert public and unfortunately the scene changes, it is impossible that a no-expert public could constitute a good and complete training data in order to update the system.

A solution in the continuity of works of Zhou et al. [12] and Senior et al. [9] is proposed. Indeed, 6] need the depth information of the object in the scene. So they need to use stereo triangulation. But, like in figure 1, this system is composed of two almost collocated cameras.

Moreover, in the goal of an automatic and autonomous system, the solution of Jain et al. [6] and Senior et al. 9] are not used. In fact, they need an expert person knowing to use a calibration marks in the case of Jain et al. [6] or knowing to extract the good datas to make the training datas in the case of Senior et al. 9].

In this paper, an automatic and autonomous solution is presented for a nocalibrated pair of static-dynamic cameras. The solution adapts automatically to its environment. In fact, if the pair of cameras are in a changing environment, this solution can be restarted regularly.

\section{Automatic Supervised Multi-sensor Calibration Method}

The system used is composed of a static camera with a wide field of view and a Pan-Tilt-Zoom camera with a field-of-view 2.5 times smaller than that of the static camera at the minimal zoom. In the following, the images of the static and the PTZ camera are respectively noted $I_{s}$ and $I_{d}(\alpha, \beta, Z)$. The parameters $(\alpha, \beta, Z)$ represent the pan, tilt and zoom parameters of the PTZ camera. 
The method presented in this section enables to learn the relation $\zeta$, for all zoom $Z_{j}$, between the pixel coordinates $\left(x_{s}, y_{s}\right)$ of $I_{s}$ and the pan-tilt parameters depending of the zoom $Z_{j}$ :

$$
\left(\alpha_{Z_{j}}, \beta_{Z_{j}}\right)=\zeta\left(x_{s}, y_{s}, Z_{j}\right) .
$$

To learn the relation $\zeta$, two steps are needed. The first step is the computation of an camera-to-camera mapping (LUT). The LUT gives the relation between $n_{s}$ pre-defined pixels of $I_{s}$ and the pan-tilt parameters such as the pixel is mapped to the center $C_{d}$ of $I_{d}(\alpha, \beta, Z)$ for different samples of the zoom $Z_{j=0,10, \ldots, m}$. In the following, the $n_{s}$ pre-defined pixels of $I_{s}$ are called nodes and noted $\mathbf{N}$ $=\left\{N_{s}^{0}, N_{s}^{1}, \ldots, N_{s}^{n_{s}-1}\right\}$. The second step is the extension of the LUT for all the pixel of $I_{s}$ and all values of the zoom $Z$.

\subsection{Camera-to-Camera Calibration : 3D Scene Constraints Integration in LUT Computation}

Computation of the LUT. The computation of the LUT integrates two loops: (1) computation of the LUT for a constant zoom $Z_{0}$ for all the nodes of $\mathbf{N}$ and (2) computation of the LUT for each zoom $Z_{j=0,1, \ldots, m}$ for all the nodes of $\mathbf{N}$.

To begin the computation of the LUT at the zoom $Z_{0}$, we need to be in the neighbourhood $V_{0}$ of $N_{s}^{0}$. In order to move automatically the PTZ camera in $V_{0}$, pan-tilt parameters are randomly selected until the field-of-view of the PTZ is in a good neighbourhood of $N_{s}^{0}$.

The main steps of this procedure are :

1. Initialization on $N_{s}^{0}$;

2. For each node $N_{s}^{i}$ in the static camera :

(a) Selection of images $I_{s}$ and $I_{d}\left(\alpha, \beta, Z_{0}\right)$ to be compared

(b) Extraction and robust matching of interest points between $I_{s}^{\prime}$ and $I_{d}(\alpha$, $\left.\beta, Z_{0}\right)$

(c) Computation of an homography $H$ between interest points of $I_{s}^{\prime}$ and $I_{d}\left(\alpha, \beta, Z_{0}\right)$

(d) Computation of the $N_{i, s}$ coordinates in $I_{d}\left(\alpha, \beta, Z_{0}\right): N_{d}^{i}=H \times N_{s}^{i}$

(e) Command of the dynamic camera in order to $N_{d}^{i}$ catch up with $C_{d}$

(f) Process $N_{i, s}$ until the condition $\left|N_{i, d}-C_{d}\right|<\epsilon$ is reached. Otherwise we stop the loop after $k$ loops

3. Go to the step (2) to process the node $N_{s}^{i+1}$;

At the step (2a), a small image $I_{s}^{\prime}$ is extracted from the complete image $I_{s}$ around the node $N_{s}^{i}$ to process in order to optimize the matching result. In fact, the field-of-view of the PTZ camera is smaller than the one of the static camera. So, the size of $I_{s}^{\prime}$ is defined such as the field-of-view of $I_{s}^{\prime}$ is nearly the same that the field-of-view of the PTZ camera.

For the step (2b), the scale-invariant feature transform (SIFT) method proposed by Lowe 7 for extracting and matching distinctive features from images of static and PTZ cameras is used. The features are invariant to image scale, 
rotation, and partially invariant to changing viewpoints, and change in illumination.

At the step (2c), we assume that locally the area in the static and PTZ cameras can be approximated by a plane. Locally, the distortion in $I_{s}$ can be considered insignificant. So, the homography $H$ is searched such as it is the homography that best matches a set of points extracts of the lists of points obtained previously. The set of correspondences contains a lot of outliers. So, the homography $H$ is robustly estimated with a RANSAC procedure. A homography is randomly computed from only four points, and test, how many other points satisfy it many times. The optimal solution is the homography which has the highest number of good points.

When the coordinates of $N_{s}^{i}$ in $I_{d}$ are known, the parameters $(\alpha, \beta)$ of the PTZ camera must be estimated in order to insure the convergence of $N_{d}^{i}$ to the center $C_{d}$. We use a proportional controller based on the error between the coordinates of $N_{d}^{i}$ and the coordinates of $C_{d}$, such as it minimizes the criterion of the step (2e). We assume that the pan-tilt axes and the coordinates axes are collocated. So we can write :

$$
\left(\begin{array}{c}
\alpha \\
\beta
\end{array}\right)=\left(\begin{array}{cc}
K_{x \rightarrow \alpha} & 0 \\
0 & K_{y \rightarrow \beta}
\end{array}\right)\left(\begin{array}{c}
\Delta x \\
\Delta y
\end{array}\right)
$$

The error $(\Delta x, \Delta y)$ corresponds to the coordinates of $N_{i, d}-C_{d}$. As we are in a static training problem, a proportional controller is sufficient.

This procedure is repeated as long as $\left|N_{d}^{i}-C_{d}\right|<\epsilon$ is not achieved. If the system diverges, we stop after $k$ loops.

After convergence, to steer the PTZ camera in the neighbourhood of the next point $N_{s}^{i+1}$, the needed angles are estimated with the knowledge of the previous learned points. For a new point $N_{s}^{i+1}$, we search the closest point among the previous processed points for each direction. The pan-tilt parameters of the best result are used to move the PTZ camera in the neighbourhood of $N_{s}^{i+1}$.

For the computation of the LUT for the other zooms, the same procedure is used. For a given zoom $Z_{j}$, instead of comparing a small image of $I_{s}$ with an image $I_{d}$ for a node $N_{s}^{i}$, an image $I_{d}$ at the zoom $Z_{j-1}$ centerred on the node $N_{s}^{i}$ is used as the reference image for the visual servoing of the PTZ camera on the node $N_{s}^{i}$ at the zoom $Z_{j}$.

Construction of $\mathbf{N}$. The choice of the $n_{s}$ nodes $N_{s}^{i}$ depends on the information contained in the $3 \mathrm{D}$ scene. For an image $I_{s}$, interest points are extracted with SIFT method. For each pixel of $I_{s}$, we compute the density function of the interest points with the Parzen window method. The size of the window depends on the relation between each field-of-view of cameras. Then, we search the pixel in $I_{s}$ with the maximal probability : it is the first node $N_{s}^{0}$ of $\mathbf{N}$. The value zero is given to the probability of the pixels around $N_{s}^{0}$ in order to obtain a best repartition of nodes. This procedure is repeated until the last pixel of $I_{s}$ with a no-zero probability. The figure 2 shows the nodes obtained with this procedure. 

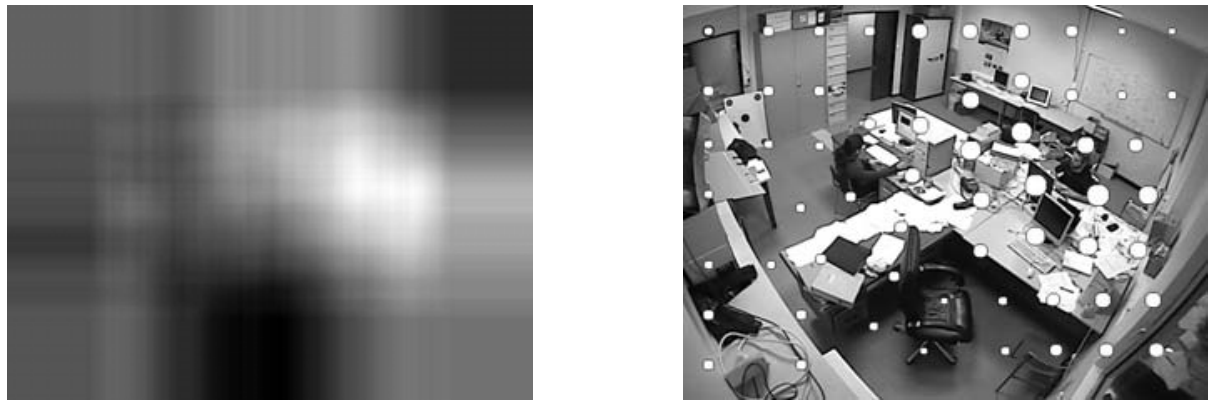

Fig. 2. The left figure represents the estimation of the density function of the interest points in $I_{s}$ by using the Parzen window method. The right figure shows an example of the extracted grid to $I_{s}$, more the circle is important more the density function is important.

\subsection{Expansion of LUT}

After the previous section 2.1 we obtain a LUT for $n_{s}$ pixels of the static image $I_{s}$ for $m$ different zooms. In order to complete the LUT for all the pixels of $I_{s}$ and all values of the zoom, an approximation of this data is searched.

In such interpolating problems, Thin-Plate-Spline (TPS) interpolation, proposed by Bookstein et al. [3], is often preferred to polynomial interpolation because it gives similar results, even when using low degree polynomials and avoids Runge's phenomenon for higher degrees (oscillation between the interpolate points with a big variation). A TPS is a special function defined piecewise by polynomials.

The computation of the interpolation relation $\zeta$ resulting of the TPS method needs a training step. The correspondences between the coordinates of a pixel of $I_{s}$ for a given zoom $Z_{j}$ and the pan-tilt parameters for $Z_{j}$ learned during the
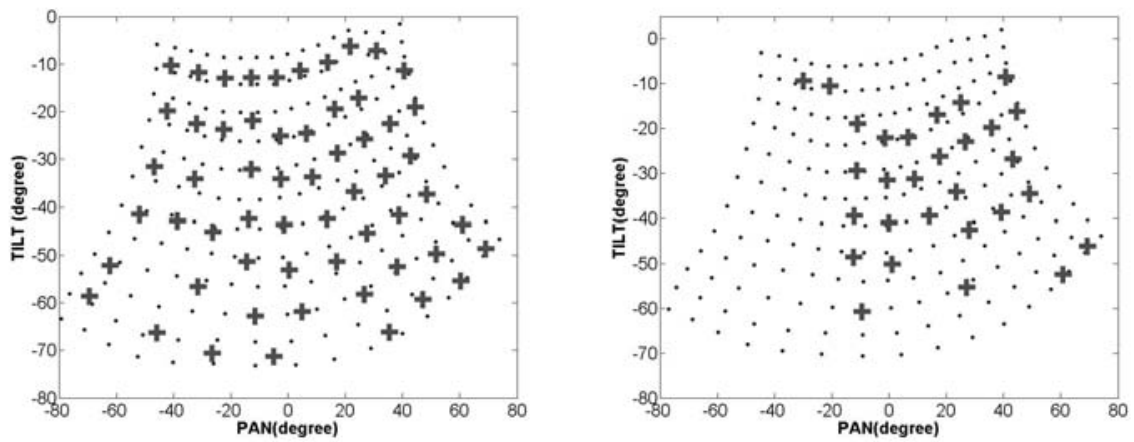

Fig. 3. Result of the TPS interpolation method : zoom $Z_{0}$ on the left figure and zoom $Z_{9}$ on the right figure. Plus correspond to the results of the computation of the LUT for the nodes of $\mathbf{N}$. Points correspond to the use of $\zeta$ for unknown pixels of $I_{s}$. 
LUT computation is used as training data for TPS method. So, for all triplet $\left(x_{s}, y_{s}, Z_{j}\right)$, the pan-tilt parameters can be estimate with $\zeta$, see figure 3 .

\section{$3 \quad$ Results and Experiments}

Cameras of the AXIS company are used. The image resolution used is $640 \times 480$ pixels for the static camera and $704 \times 576$ pixels for the PTZ camera. The field of view of the static camera is around $90^{\circ}$. In the case of minimal zoom, the field of view of the PTZ camera is of $42^{\circ}$.

The PTZ camera has a 26x optical zoom. The difference of the field of view between the two cameras can be important. For example, the field of view of the PTZ camera is 2.5 times smaller than one of the static camera at the zoom $Z_{0}$, 5 times smaller at the zoom $Z_{4}$ and 12.5 times at the zoom $Z_{7}$.

The mechanical step of the PTZ camera is $0.11^{\circ}$. Experimentally, we show that the mean displacement in the image $I_{d}$ for the minimal mechanical step depends on the zoom, see table 1] At best, the accuracy of the solution is limited by this mechanical factor.

Table 1. Mean displacement in pixel in the image of the PTZ camera for different zooms

\begin{tabular}{|c|c|c|c|c|c|c|c|c|c|}
\hline Zoom & 0 & 1 & 2 & 3 & 4 & 5 & 6 & 7 & 8 \\
\hline mean in X (pixels) & 1.64 & 2.03 & 2.42 & 2.59 & 3.75 & 4.76 & 6.93 & 9.47 & 15.71 \\
\hline mean in Y (pixels) & 1.57 & 2.99 & 2.48 & 3.54 & 5.03 & 5.43 & 7.92 & 10.46 & 13.97 \\
\hline
\end{tabular}

In order to estimate the accuracy of this supervised calibration method, it is necessary to know exactly the coordinates of a reference pixel $P_{s}$ in $I_{s}$ and to find precisely its coordinates in $I_{d}$. To solve this problem, a black ellipsis $E$ which is visible in the two cases is used. To determine with accuracy the coordinates of the center of $E$, the binarization method of Otsu [8] is used. Pixels of a region of interest can be separated in two classes. Then, the coordinates of the center of gravity of black pixels are estimated with subpixelic precision.

\subsection{Accuracy of the Visual Servoing Loop}

For evaluating the accuracy of the visual servoing loop (see section 2.1) at the zoom $Z_{0}$, three positions of the ellipsis $E$ are choosen : (1) $E$ on a node issued of the Parzen window method with a high range, (2) a node with a middle range and (3) a node with a small range.

The result of the experimentation is given on the figure 4 with the absolute errors of pan and tilt. For the case 1, the standard deviation is small and of the same order of the mechanical step $\left(0.11^{\circ}\right)$. For the other two cases, the number of interest points around the nodes is less important so the error and the standard deviation increase. The estimation of the pan-tilt parameters is less accuracy. This result shows that it is important to choose an to classify the nodes in function of the density of interest points around the nodes. 

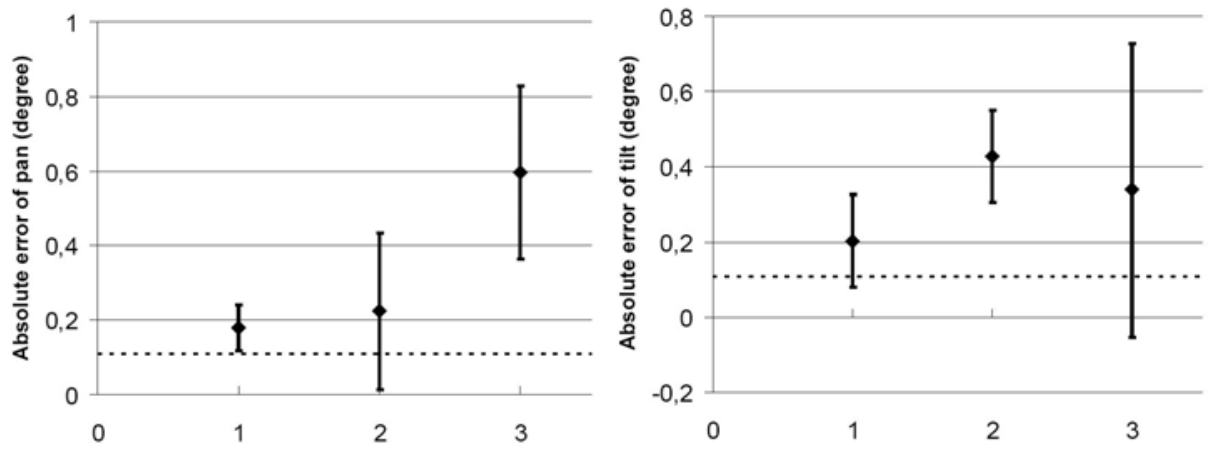

Fig. 4. Absolute errors of pan and tilt in degree resulting of the visual servoing loop for three positions $\mathrm{n}$ the scene. The dot line represents the mechanical step.

\subsection{Accuracy of the Supervised Calibration Method}

For evaluate the accuracy of the complete solution (see section 21), three cases are choosen : (1) $E$ on a pixel which is on the same 3D plane that the closer learned nodes, (2) $E$ on a pixel which is on a different 3D plane that each closer learned node and (3) $E$ on an unknow object when $\zeta$ is learned, see figure 5 .

The result of the experimentation is given on the figure 6 with the error normalized to mechanical step for the parameters pan-tilt. The case 1 (triangle) is the most ideal case because the $3 \mathrm{D}$ information is homogeneous. So, the error is small. The case 2 (diamond) is more complex because the $3 \mathrm{D}$ information of the scene presents big variations. So, the error is bigger than the case 1 . In the case 3 (square), the unknown object modify the geometry of the scene. This variation was not learned during the learning step of $\zeta$. So the error is more important than the previous cases. Moreover, we note on the figure 6 the error increases with the zoom. Along the learning of the LUT for several zoom, the
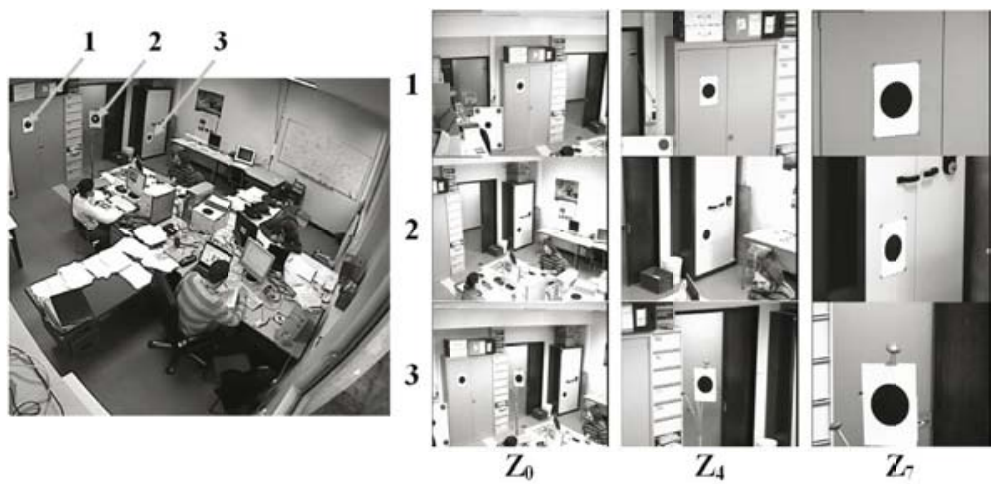

Fig. 5. Illustration of the method. The left figure represents the static camera with the three positions of the target noted. On the right figure, the result for the PTZ camera is shown for different levels of zoom. 

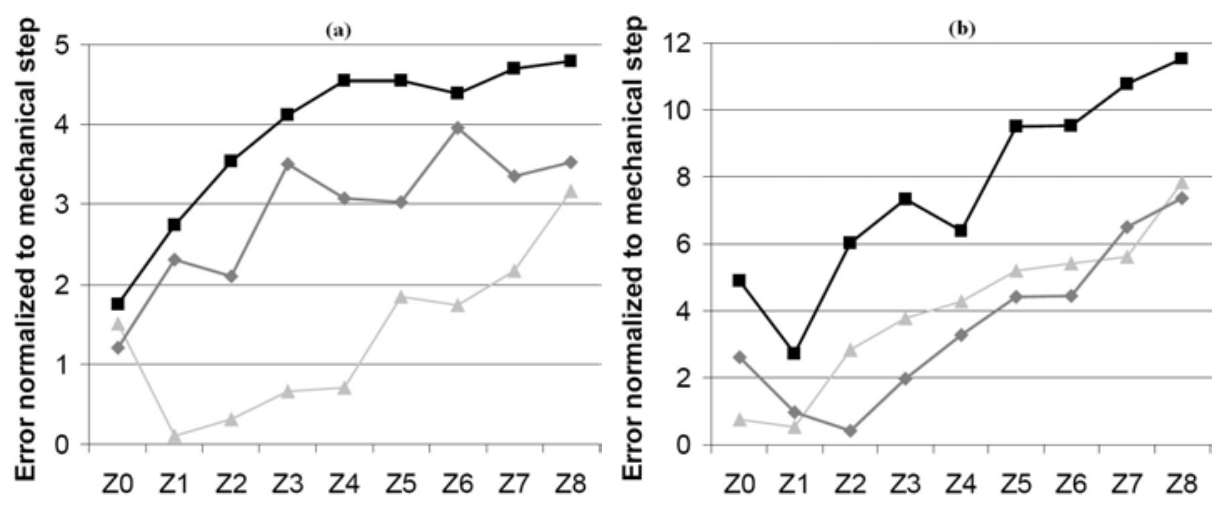

Fig. 6. Error normalized to mechanical step for pan (a) and tilt (b) parameters for several zoom for three cases in the 3D scene (see figure 5) : triangle for case 1, diamond for case 2 and square for case 3

comparison is made between two consecutive zooms. So, the error accumulates progressively. But, the figure 5 shows that even for high zoom, the result can be a good initialization to track a person.

\section{Conclusion and Perspectives}

In this paper, an automatic algorithm of camera-to-camera calibration integrating the zoom calibration was presented in order to steer a PTZ camera using information of the static camera. At the end, we obtain the relation $\zeta$, for all zoom $Z_{j}$, between the pixel coordinates $\left(x_{s}, y_{s}\right)$ of $I_{s}$ and the pan-tilt parameters depending on the zoom $Z_{j}:\left(\alpha_{Z_{j}}, \beta_{Z_{j}}\right)=\zeta\left(x_{s}, y_{s}, Z_{j}\right)$.

All the parameters are automatically selected. The process includes a measure of grey level activity (SIFT). We want to apply the system with an automatic reconfiguration to develop a tracking survey system to focus on the human face along a sequence of displacement.

In the future, in order to reduce the error resulting from the step of the LUT learning for several levels of zoom, the envisaged solution is to compare the zoom $Z_{j}$ with the zoom $Z_{0}$ and to change the zoom $Z_{0}$ for a high zoom when the difference between the zoom $Z_{j}$ and $Z_{0}$ is too important. Then, after this amelioration, the solution will be tested in real condition of people tracking.

\section{References}

1. Barreto, J.P., Peixoto, P., Batista, J., Araujo, H.: Tracking Multiple Objects in 3D. IEEE Intelligent Robots and Systems. IEEE Computer Society Press, Los Alamitos (1999)

2. Basu, A., Ravi, K.: Active camera calibration using pan, tilt and roll. IEEE Transactions on Systems Man and Cybernetics. IEEE Computer Society Press, Los Alamitos (1997) 
3. Bookstein, F.L.: Principal Warps: Thin-Plate Splines and the Decomposition of Deformations. IEEE Transactions on Pattern Analysis and Machine Intelligence. IEEE Computer Society Press, Los Alamitos (1989)

4. Davis, J., Chen, X.: Calibrating pan-tilt cameras in wide-area surveillance networks. ICCV '03: Proceedings of the Ninth IEEE International Conference on Computer Vision, p. 144. IEEE Computer Society, Washington (2003)

5. Horaud, R., Knossow, D., Michaelis, M.: Camera cooperation for achieving visual attention. Machine Vision Application, vol. 16, pp. 1-2. Springer, Heidelberg (2006)

6. Jain, A., Kopell, D., Kakligian, K., Wang, Y.-F.: Using Stationary-Dynamic Camera Assemblies for Wide-area Video Surveillance and Selective Attention. IEEE Computer Vision and Pattern Recognition, pp. 537-544. IEEE Computer Society, Los Alamitos (2006)

7. Lowe, D.G.: Object Recognition from Local Scale-Invariant Features. ICCV '99: Proceedings of the International Conference on Computer Vision, vol. 2, p. 1150. IEEE Computer Society, Washington (1999)

8. Otsu, N.: A threshold selection method from grey scale histogram. IEEE Transactions on Systems Man and Cybernetics, vol. 1, pp. 62-66. IEEE Computer Society Press, Los Alamitos (1979)

9. Senior, A.W., Hampapur, A., Lu, M.: Acquiring Multi-Scale Images by Pan-TiltZoom Control and Automatic Multi-Camera Calibration. WACV-MOTION '05: Proceedings of the Seventh IEEE Workshops on Application of Computer Vision, vol. 1, pp. 433-438. IEEE Computer Society, Washington (2005)

10. Shih, S., Hung, Y., Lin, W.: Calibration of an active binocular head. IEEE Transactions on Systems Man and Cybernetics. IEEE Computer Society Press, Los Alamitos (1998)

11. Woo, D.C., Capson, D.W.: 3D visual tracking using a network of low-cost pan/tilt cameras. canadian Conference on Electrical and Computer Engineering Conference Proceedings. IEEE Computer Society Press, Los Alamitos (2000)

12. Zhou, X., Collins, R.T., Kanade, T., Metes, P.: A master-slave system to acquire biometric imagery of humans at distance. IWVS '03: First ACM SIGMM international workshop on Video surveillance, pp. 113-120. ACM Press, New York (2003) 